\title{
Numerical modeling of automated tape placement process of unidirectional thermoplastic
}

\author{
Aleksandr N. Anoshkin ${ }^{1}$, Pavel V. Pisarev ${ }^{1}$, and Yulia S. Kirova ${ }^{1, *}$ \\ ${ }^{1}$ Perm National Reserch Polytechnical University, Perm, Russia
}

\begin{abstract}
Within the framework of the present work, studies are being carried out to select the tooling material for the automated placement of the thermoplastic prepreg. A mathematical model of the heating of the tooling and a thermoplastic prepreg tape with an automated placement of the prepreg on the mandrel is developed. In the framework of numerical experiments, two rigs made of steel and carbon fiber were considered. Based on the results of numerical experiments, the temperature field distributions in the laid out part and in the tooling were obtained. The analysis of the obtained results revealed that when using metal tools, a considerable heat removal from the heated layers of the thermoplastic is observed, which can significantly influence the crystallization process of the thermoplastic. Also on the carbon-fiber tooling, the laser warming spot is more localized than in the metallic one. On the basis of the data obtained, the linear deformation of the rigging was calculated, it was found that the maximum deformations in the steel tooling are three times higher than the maximum deformations in the carbon fiber. It is determined that when using metal equipment, a significant heat removal from the heated layers of the thermoplastic is observed.
\end{abstract}

\section{Introduction}

In recent years, the field of application of composite materials has increased significantly. First of all, due to their wide use in civil aircraft and engine production. The use of composites allows significantly (for a number of parts in several times) to reduce weight, improve the environmental parameters and economy of the aircraft. In recent years, there has been an increased interest in the use of composite materials with thermoplastic matrices (TCM) in the manufacture of a wide range of products, including critical parts of aviation equipment. These materials can drastically reduce the time and laboriousness of parts manufacturing, since they do not require a polymerization and curing reaction (duration from 4 to 12 hours) [1]. In addition, thermoplastic composites have other advantages: unlimited shelf life of the semi-finished product, the ability to relax stresses, increased fracture toughness and shock resistance, fatigue endurance, the possibility of recycling, explosion safety, non-toxicity. Also, TCM are used to create aviation equipment, where the

\footnotetext{
${ }^{*}$ Corresponding author: kirova.yulia@yandex.ru
} 
operating temperature is outside the high-temperature epoxy resins, using the important advantage of TCM - thermal stability $[2,3]$. Thus, thermoplastics can provide potentially more significant savings in production and high operational properties of aviation equipment. For example, in the development of Airbus A340-600 and A380 liners were used thermoplastic shells, which weigh $20 \%$ less than comparable aluminum [4].

There are manual and automated methods for processing thermoplastic prepregs. Automated methods are divided into: Automated Tape Laying (ATL); Automated Fiber Placement (AFP) [5]. In this paper, the ATL method is considered. With this method of manufacturing parts from a polymer composite material on the basis of thermoplastic binders, the prepreg tape is laid out or wound onto a preformed forming tool with an automatic robotic complex. The method has received a lively interest in recent years because of the ability to produce products without autoclaving [6]. During the laying, the prepreg band is heated by a laser beam in the melt temperature range of the thermoplastic binder with the welding of each subsequent prepreg layers formed as a result of the laying to the previous ones. Sealing of layers of thermoplastic prepreg is carried out by way of a laying-clamping device, for example, a pressure roller. Further, the layers obtained are cooled naturally, or by directional airflow, with a reduced temperature (Fig. 1).

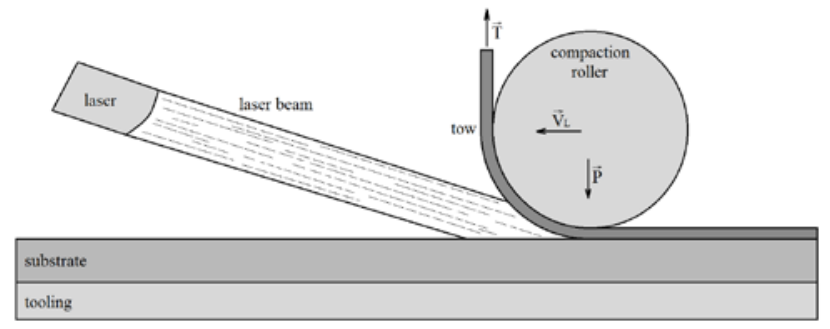

Fig. 1. Scheme of the process of automated tape placement

With the automated laying of the thermoplastic prepreg, there is a problem of selecting the parameters of the technological process: the tension of the belt, the temperature in the area of the tape contact with the tool, the force of pressing the tape to the tooling, and the choice of the tooling material. One of the important parameters of the ATL process is the selection of the tooling material. Since, the great influence on the physico-mechanical properties of the thermoplastic being processed, the thermal conductivity of the material of the tooling on which the strip of material is made [7] has an effect. Tooling for the manufacture of products from composite materials ensures their quality and performance. At the moment, in the production of aviation equipment, the most widely used equipment is steel, aluminum, for particularly critical parts, the Invar is used. There is also a trend of transition from metal rigging to carbon fiber plastic, which allows to produce large-sized products with minimal technological costs. When designing and manufacturing equipment from carbon plastic, it is necessary to take into account a number of requirements for the material: temperature resistance, resistance to stresses and deformations, ensuring uniform preheating and cooling of the prepreg. To ensure the formulated requirements, there is a need to perform numerical modeling on the evaluation of thermal fields on the surface, as well as in the layers of the thermoplastic prepreg tape.

\section{Numerical simulation}

As the object of study, a band of thermoplastic prepreg, made of unidirectional CM (fiber content $58 \%$,) is considered. Geometric dimensions of the tape: $300 * 60 * 0.15 \mathrm{~mm}$ In the process of automated laying, the tape is placed on top of the first (supporting) thermoplastic 
layer located on the tooling. The contact area between the top and bottom layer of the prepreg strip is heated by a moving laser beam with the temperature of the contact spot on the surfaces of the prepreg tape $420^{\circ} \mathrm{C}$ (Figure 2). Simulation of the motion of the contact spot of the laser is carried out in a fixed coordinate system. The considered warm-up time in the static region of the contact spot is $0.06 \mathrm{~s}$. This time corresponds to the time of passage of the laser focal spot relative to the point, at a laying speed of $1 \mathrm{~m} / \mathrm{s}$. In the framework of computational experiments, two accessories of rectangular shape $600 * 300 * 5$ $\mathrm{mm}$, made of steel and carbon fiber, are considered. The problem of numerical calculation of temperature fields $T(x, y, z, t)$ in tapes of prepreg and tooling is posed. Processes are considered three-dimensional, non-stationary, because the heat exchange between the prepreg layers and the equipment depends on the depth of pre-preheating. To simulate the heating of layered $\mathrm{CM}$ with a thermoplastic matrix in an automated production, a geometric model was developed and constructed (Fig. 2).

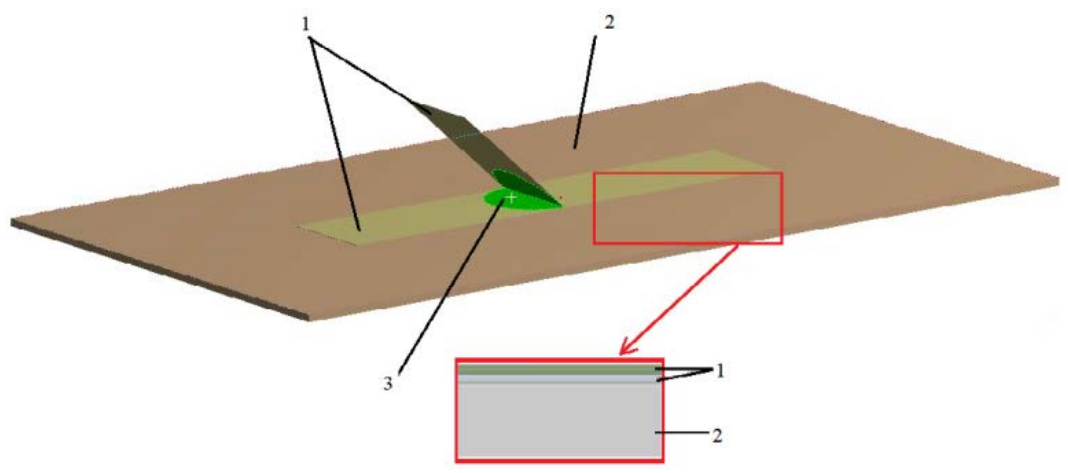

Fig. 2. General view of the geometric model where: 1-belt prepreg; 2 - rigging; 3 - focal laser spot

The mathematical formulation of the problem includes the nonstationary heat equation for anisotropic bodies [8].

$$
\begin{aligned}
& C_{p} \rho \frac{\partial T}{\partial t}=\lambda_{11}\left(\frac{\partial T}{\partial x}\right)^{2}+\lambda_{22}\left(\frac{\partial T}{\partial y}\right)^{2}+\lambda_{33}\left(\frac{\partial T}{\partial z}\right)^{2}+\left(\lambda_{23}+\lambda_{32}\right) \frac{\partial T}{\partial y} \frac{\partial T}{\partial z}+\left(\lambda_{31}+\lambda_{13}\right) \frac{\partial T}{\partial z} \frac{\partial T}{\partial x}+ \\
& +\left(\lambda_{12}+\lambda_{21}\right) \frac{\partial T}{\partial x} \frac{\partial T}{\partial y}
\end{aligned}
$$

where $T(x)$ is the value of the temperature field; $\lambda_{11}, \lambda_{22}, \lambda_{33}, \lambda_{23}, \lambda_{32}, \lambda_{31}, \lambda_{13}, \lambda_{12}, \lambda_{21^{-}}$ thermal conductivity of the material in the $x, y, z ; t$-time; $C_{p}$ - is the specific heat of the material; $\rho$ - the density of the material.

The boundary conditions for the problem under consideration were formulated as follows: 1) initial conditions for the thermoplastic, tooling and external environment temperature $T$ $\left.(x, y, z, 0)=22^{\circ} \mathrm{C} ; 2\right)$ At the point of contact between the layers and in the region of interaction between the lower layer and the rigging, boundary conditions of the fourth kind were assigned (2); 3) A contact spot with a constant heating temperature $T(x, y, z, t)=$ $420^{\circ} \mathrm{C}$ is placed between the lower face of the upper layer and the upper face of the lower layer of the thermoplastic; Geometric characteristics of the focal spot were determined from the thermal imaging control data obtained for the automatic method of manufacturing thermoplastics (Fig. 3) [9]. In the contact region of the upper face of the upper layer of the prepreg with the external medium, the upper face of the lower layer of the prepreg with the external medium and the lower face of the tooling with the external medium, boundary conditions of the third kind (3) 


$$
\lambda_{1} \frac{\partial T_{1}(t)}{\partial n} \overline{l_{n}}=\lambda_{2} \frac{\partial T_{2}(t)}{\partial n} \overline{l_{n}}
$$

where $\lambda_{1}, \lambda_{2}$-coefficients of thermal conductivity of the first and second body; $T_{1}, T_{2}-$ the temperature of the first and second body at the interface of their contact; $\bar{l}_{n}$ - normal to the surface of the body.

$$
\lambda \frac{\partial T}{\partial n}=h\left(T_{n}(M, t)-T_{o c}\right)
$$

where $T_{n}$ - thermoplastic layer temperature value; $T_{o c}$ - value of ambient temperature; $h$ coefficient of heat exchange between the medium and the surface of the body; $\mathrm{M}$ - point belonging to the surface; $l_{n}$ - normal to the surface of the body.

The thermophysical properties of the anisotropic layers of the thermoplastic prepreg were determined analytically based on the properties and relationships presented in [10].
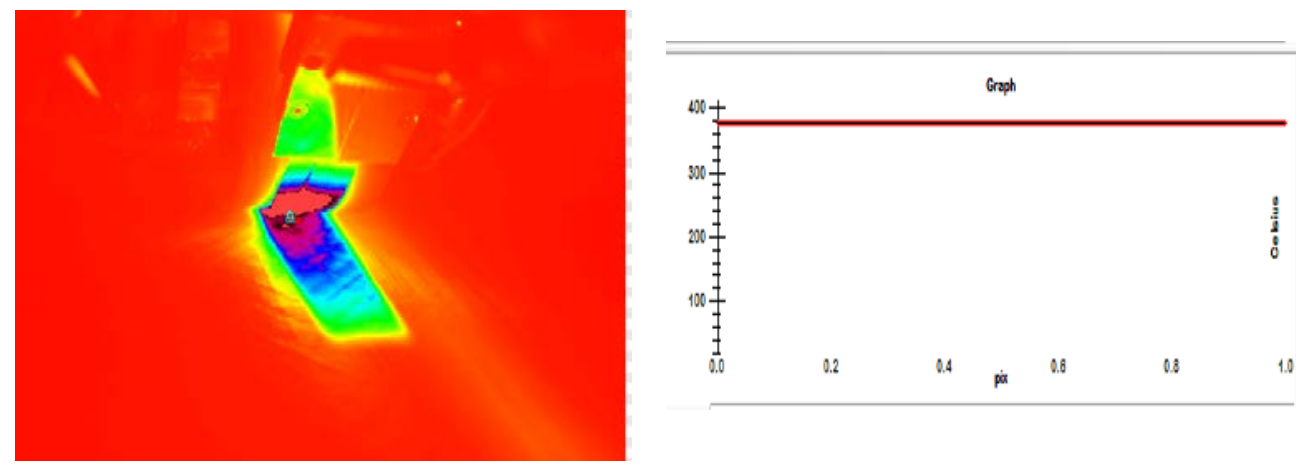

Fig. 3. Results of thermal imaging control obtained for the automatic method of manufacturing thermoplastics [9]

During the construction and local grinding of the grid, sharp differences in the geometric dimensions of the neighboring elements (more than 2 times) were not allowed. Adaptation of the grid was carried out in the regions of high stress gradients, namely on the surface of the input and output edges. The maximum element size for the prepreg tape was $1 \mathrm{~mm}$, for the tooling $2 \mathrm{~mm}$. (Fig. 4). The total number of finite elements was 2000 thousand elements. The final elements of the tooling are hexagonal in shape. The elements of the prepreg tape represent the tetrahedral shape.

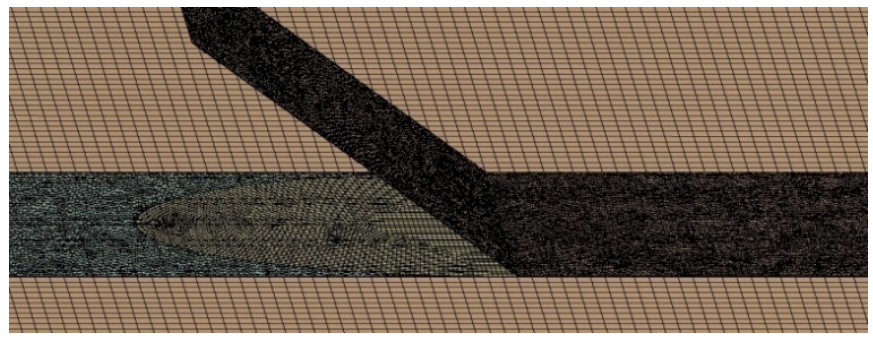

Fig. 4. General view of the finite element model of a laminated workpiece on a flat tooling during automated manufacturing

As a method of solution, the finite element method is chosen. The numerical model is implemented in the ANSYS Workbench software package. 
Based on the results of computational experiments, in the steady-state regime, the temperature distribution fields in the prepreg bands and in the tooling material were obtained. Figure $5 \mathrm{a}, \mathrm{b}$ shows the temperature fields along the surface of the upper and lower layer of the thermoplastic located on the metal (a) and carbon-plastic (b) fittings. Figure 6 shows the temperature distribution fields over the surface of the metallic (a) and carbon-plastic (b) tooling.
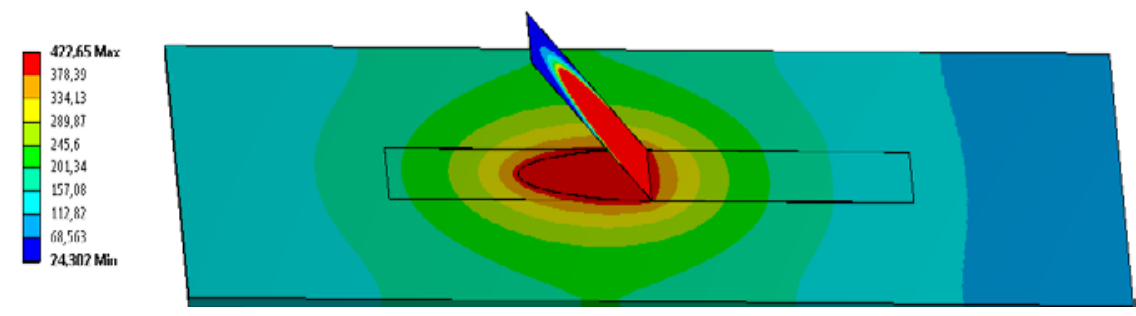

a
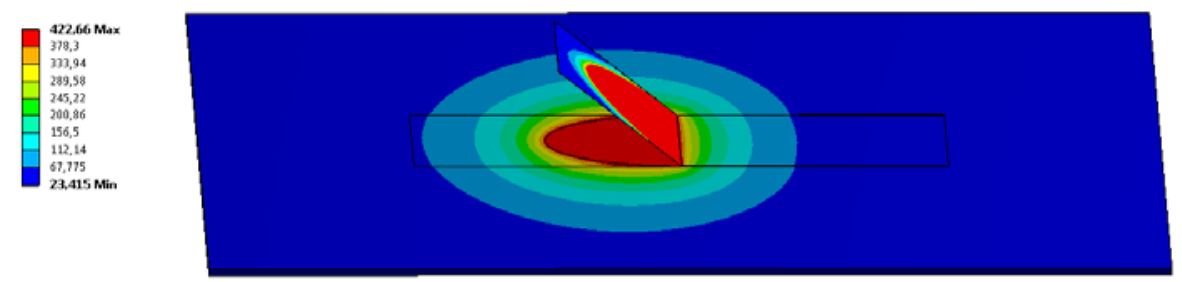

b

Fig. 5. Temperature distribution over the surface of the thermoplastic tape located on the tooling
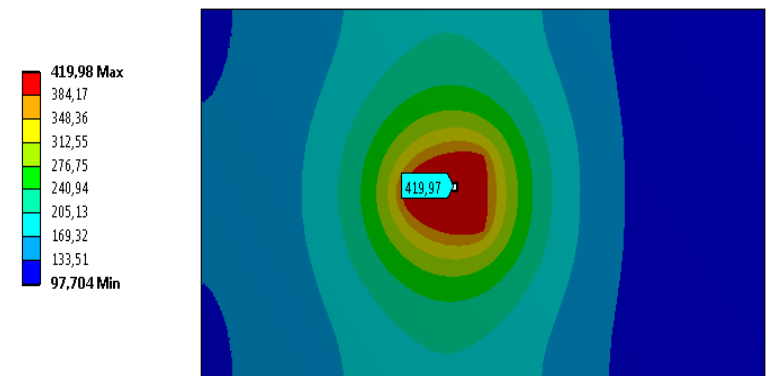

a

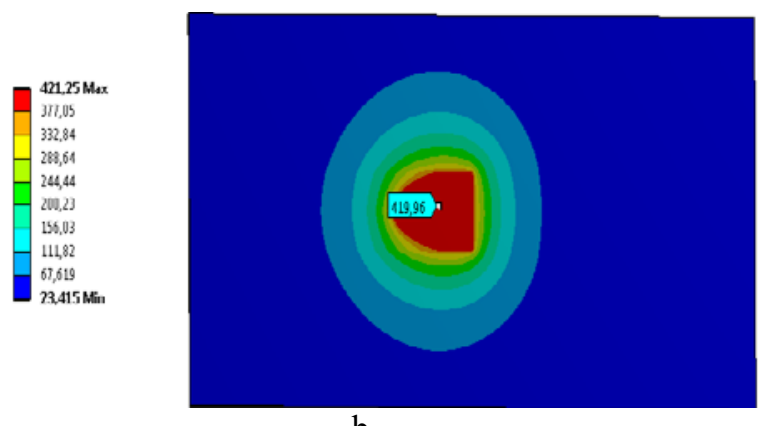

b

Fig. 6. Temperature distribution over the surface of the tooling 
The analysis of the obtained results revealed that when using metal equipment, considerable heat removal from the heated layers of the thermoplastic is observed. It is determined that for the carbon-fiber tooling the heating region does not exceed three characteristic sizes of the contact spot. The results of the maximum and minimum temperature values on the surface of the snap are shown in Table 1.

Table 1. Temperature values for two types of equipment

\begin{tabular}{|l|l|l|}
\hline Type of tooling & $\begin{array}{l}\text { Maximum temperature } \\
\text { value, }{ }^{\circ} \mathbf{C}\end{array}$ & $\begin{array}{l}\text { Minimum temperature } \\
\text { value, }{ }^{\circ} \mathbf{C}\end{array}$ \\
\hline Metallic & 419 & 97,7 \\
\hline Carbon-plastic & 419 & 23,4 \\
\hline
\end{tabular}

Analyzing the results presented in Figures 5 and 6 , it can be noted that at the maximum exposure time of a laser source with a constant temperature, the distribution of heat in the carbon fiber tooling is most localized, in comparison with the metallic one. From which it was concluded that the thermoplastic layers will be heated more effectively when using a tool made of carbon fiber. However, when the laser source is exposed to a long-term effect in the local area, the temperature in the carbon fiber tool can exceed the temperatureresistance limit of the material.

Based on the results of computational experiments in non-stationary formulations, the temperature distribution fields in the prepreg and the tooling tapes for the time instant $\mathrm{t}=$ $0.06 \mathrm{~s}$. were obtained. Figure $7 \mathrm{a}, \mathrm{b}$ shows the distribution of temperature in the longitudinal section of the upper and lower layers of the thermoplastic. Figure 8 shows the temperature distribution fields over the surface of the metallic (a) and carbon-plastic (b) tooling at the time $\mathrm{t}=0.06 \mathrm{~s}$.
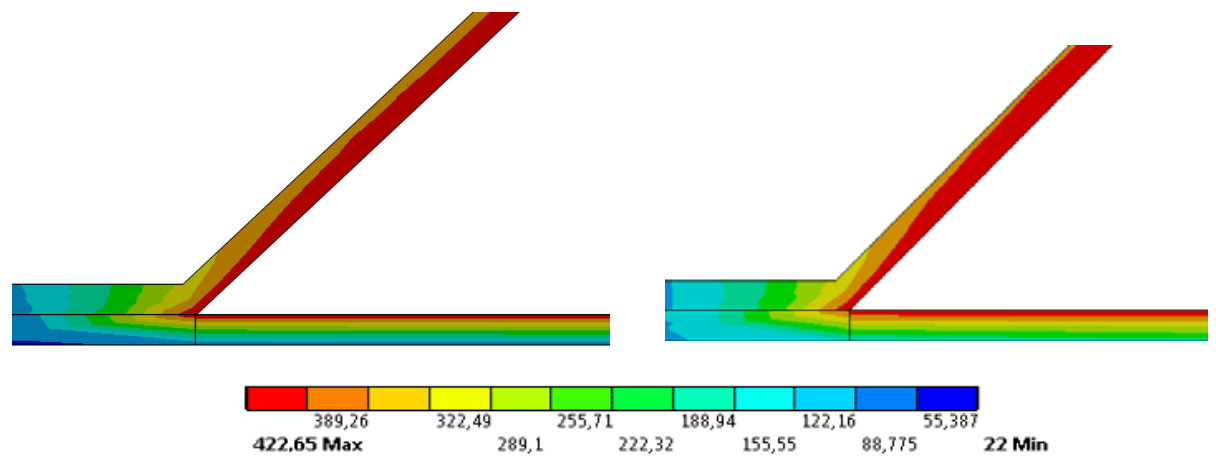

a

b

Fig. 7. Temperature distribution in the longitudinal section of two layers of a thermoplastic for a metallic, $b$ - carbon-plastic equipment

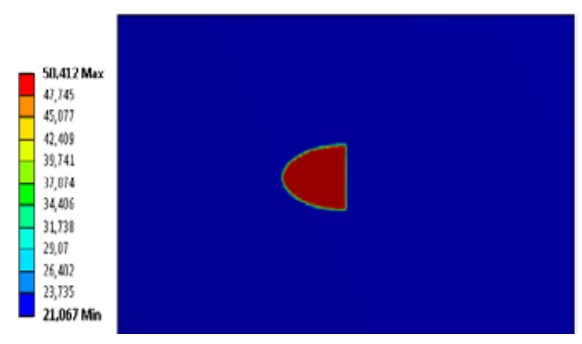

a

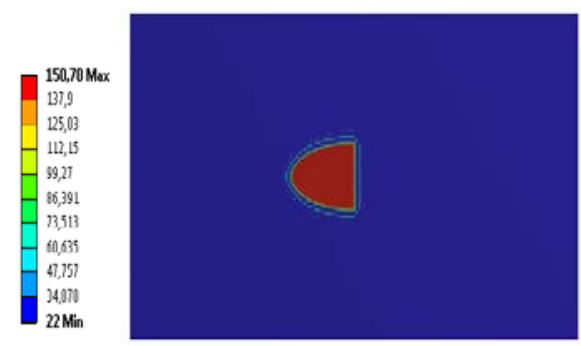

b

Fig. 8. Temperature distribution over the tooling at time $t=0.06 \mathrm{~s}$. 
The analysis of the obtained results revealed that when using metal equipment, considerable heat removal from the heated layers of the thermoplastic is observed. The results of the maximum heating temperature of the tooling and the warming-up depth of the tape are shown in Table 2.

Table 2. Temperature values and depth of heating at time $t=0.06 \mathrm{~s}$.

\begin{tabular}{|l|l|l|}
\hline Type of tooling & $\begin{array}{l}\text { Maximum temperature } \\
\text { value, }{ }^{\circ} \mathbf{C}\end{array}$ & $\begin{array}{l}\text { The depth of heating of the } \\
\text { second layer to the bonding } \\
\text { temperature }\left(\mathbf{4 0 0}{ }^{\circ} \mathbf{C}\right), \mathbf{m m}\end{array}$ \\
\hline Metallic & 50,412 & 0,02 \\
\hline Carbon-plastic & 150 & 0,035 \\
\hline
\end{tabular}

Based on the data obtained in Table 2, a linear deformation was calculated for a rectangular tooling. At the same time, based on the maximum value of temperature, the value of linear deformation for steel $15,3 \cdot 10^{-6} \mathrm{grad}^{-1}$, carbon-plastic $10^{-6} \mathrm{grad}^{-1}$ was adopted.Linear deformations were obtained from the known relation $\varepsilon=\Delta \mathrm{T} \alpha$. For the metal equipment, the value is $0.42 \%$, for the carbon-plastic is $0.13 \%$.

\section{Conclusion}

Within the framework of the research, a mathematical model has been developed that describes the non-stationary processes of heating the tooling and tape of a thermoplastic prepreg in the process of automated stacking. Based on the results of numerical experiments, the temperature field distributions in the laid out part and in the tooling were obtained. The analysis of the obtained results revealed that when using metal equipment, considerable heat removal from the heated layers of the thermoplastic is observed. Based on the simulation results, it can be said that the temperature of the tooling can influence the temperature of the prepreg and, consequently, affect the degree of crystallization of the matrix. The crystallization task is rather laborious and requires further research. On the basis of the data obtained, the linear deformation of the rigging was calculated, it was found that the maximum deformations in the steel tooling are three times higher than the maximum deformations in the carbon fiber. On the basis of the conducted researches it is possible to draw a conclusion that it is possible to make products from TCM on carbonfiber tooling, since less linear deformations are obtained and the material is more technological. Also, based on the results of the simulation, the tooling for the automated layering of the prepreg should be made of carbon fiber reinforced plastic on a highly resistant temperature binder.

The work was carried out with the financial support of the state represented by the Ministry of Education and Science of Russia, the unique identifier of the project RFMEFI57717X0261.

\section{References}

1. M.N. Grimshaw, C.G. Grant, J.M.L. Diaz, Advanced technology tape laying for affordable manufacturing of large composite structures, In: 46-th International SAMPE Symposium, pp. 2484-2494 (2001)

2. S. Gao, Cooling rate influences in carbon/PEEK composites, Part III: impact damage performance, Compos. A Appl. Sci. Manuf., 32, pp. 763-774 (2001)

3. J.-M. Bai, D. Leach, S. Cease, J. Pratte, High performance thermoplastic polymers and composites. In: SAMPE, Corina, CA, USA, S., pp. 1391-1405 (2005) 
4. C. Red, The Outlook for Thermoplastics in Aerospace Composites, 2014-2023, Composites World, (2014)

5. Yu. A. Gusev, A.V. Borshchev, A.V. Khrulkov, Features of prepregs for automated layering by ATL and AFP methods, Electronic Scientific Journal "VIAM WORKS", 3 (2015)

6. C. Dedieu, A. Barasinski, F. Chinesta, J.-M. Dupillier, On the prediction of residual stresses in automated tape placement, International Journal of Material Forming, 4, pp. 633-640 (2017)

7. N.A. Demkovich, I.A. Volkov, E.I. Yablochnikov, Application of numerical simulation systems for the introduction of new production technologies, Izvestiya Samara Scientific Center, Russian Academy of Sciences, vol. 18, 4 (3), pp. 459-463 (2016)

8. G. Carslow, D. Yeher, Thermal conductivity of solids (Nauka, 1964)

9. Technical and commercial offer of Mikrosam

10. B.N. Fedulov, A.A. Saonov, M.M Kantor, S.V. Lomov, Modeling of thermoplastic composites solidification and estimation of residual stresses, Composites and Nanostructures, v. 9, 2, pp. 102-122 (2017) 